\title{
Hypergeometric Functions and Carlitz Differential Equations over Function Fields
}

\author{
Anatoly N. Kochubei \\ Institute of Mathematics, \\ National Academy of Sciences of Ukraine, \\ Tereshchenkivska 3, Kiev, 01601 Ukraine \\ E-mail: kochubei@i.com.ua
}




\begin{abstract}
The paper is a survey of recent results in analysis of additive functions over function fields motivated by applications to various classes of special functions including Thakur's hypergeometric function. We consider basic notions and results of calculus, analytic theory of differential equations with Carlitz derivatives (including a counterpart of regular singularity), umbral calculus, holonomic modules over the Weyl-Carlitz ring.
\end{abstract}

2000 Mathematics Subject Classification: Primary 11S80, 12H25, 33E50. Secondary 05A40, $11 \mathrm{G} 09,16 \mathrm{~S} 32,32 \mathrm{C} 38$. 


\section{INTRODUCTION}

Let $K$ be the field of formal Laurent series $t=\sum_{j=N}^{\infty} \xi_{j} x^{j}$ with coefficients $\xi_{j}$ from the Galois field $\mathbb{F}_{q}, \xi_{N} \neq 0$ if $t \neq 0, q=p^{v}, v \in \mathbf{Z}_{+}$, where $p$ is a prime number. It is well known that any non-discrete locally compact field of characteristic $p$ is isomorphic to such $K$. The absolute value on $K$ is given by $|t|=q^{-N},|0|=0$. The ring of integers $O=\{t \in K:|t| \leq 1\}$ is compact in the topology corresponding to the metric $\operatorname{dist}(t, s)=|t-s|$. The absolute value $|\cdot|$ can be extended in a unique way onto the completion $\bar{K}_{c}$ of an algebraic closure of $K$.

Analysis over $K$ and $\bar{K}_{c}$ initiated by Carlitz [5] and developed subsequently by Wagner, Goss, Thakur, the author, and others (see the bibliography in [14, 39]) is very different from the classical calculus. The new features begin with an appropriate version of the factorial invented by Carlitz - since the usual factorial $i$ !, seen as an element of $K$, vanishes for $i \geq p$, Carlitz introduced the new one as

$$
D_{i}=[i][i-1]^{q} \ldots[1]^{q^{i-1}}, \quad[i]=x^{q^{i}}-x(i \geq 1), D_{0}=1 .
$$

An important feature is the availability of many non-trivial $\mathbb{F}_{q}$-linear functions, that is such

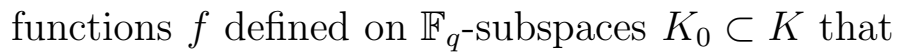

$$
f\left(t_{1}+t_{2}\right)=f\left(t_{1}\right)+f\left(t_{2}\right), \quad f(\alpha t)=\alpha f(t),
$$

for any $t, t_{1}, t_{2} \in K_{0}, \alpha \in \mathbb{F}_{q}$. Such are, for example, polynomials and power series of the form $\sum a_{k} t^{q^{k}}$, in particular, the Carlitz exponential

$$
e_{C}(t)=\sum_{n=0}^{\infty} \frac{t^{q^{n}}}{D_{n}}, \quad|t|<1,
$$

and its composition inverse, the Carlitz logarithm

$$
\log _{C}(t)=\sum_{n=0}^{\infty}(-1)^{n} \frac{t^{q^{n}}}{L_{n}}, \quad|t|<1,
$$

where $L_{n}=[n][n-1] \cdots[1](n \geq 1), L_{0}=1$. The notion of the Carlitz exponential obtained a wide generalization in the theory of Drinfeld modules (see [14, 39]). On the other hand, in various problems going beyond the class of $\mathbb{F}_{q}$-linear functions, an extended version of the Carlitz factorial (and its Gamma function interpolations) is used, so that $D_{n}$ can be seen as "an $\mathbb{F}_{q}$-linear part" of the full factorial; see [14, 39] and references therein for the details.

Among other special classes of $\mathbb{F}_{q}$-linear functions there are various polynomial systems (see below), an analog of the Bessel functions [6, 36], and Thakur's hypergeometric function [37, 38, 39]. The latter is defined as follows.

For $n \in \mathbb{Z}_{+}, a \in \mathbb{Z}$, denote

$$
(a)_{n}= \begin{cases}D_{n+a-1}^{q^{-(a-1)},}, & \text { if } a \geq 1 \\ L_{-a-n}^{-q^{n}}, & \text { if } a \leq 0, n \leq-a \\ 0, & \text { if } a \leq 0, n>-a\end{cases}
$$


Then, for $a_{i}, b_{i} \in \mathbb{Z}$, such that the series below makes sense, we set

$$
{ }_{r} F_{s}\left(a_{1}, \ldots, a_{r} ; b_{1}, \ldots, b_{s} ; z\right)=\sum_{n=0}^{\infty} \frac{\left(a_{1}\right)_{n} \cdots\left(a_{r}\right)_{n}}{\left(b_{1}\right)_{n} \cdots\left(b_{s}\right)_{n} D_{n}} z^{q^{n}} .
$$

Thakur 37, 38, 39, has carried out a thorough investigation of the functions (1.5) and obtained analogs of many properties known for the classical situation. In particular, he found an analog of the hypergeometric differential equation. Its main ingredients are the difference operator

$$
(\Delta u)(t)=u(x t)-x u(t)
$$

(an inner derivation of composition rings of $\mathbb{F}_{q}$-linear polynomials or more general $\mathbb{F}_{q}$-linear functions) introduced by Carlitz [5], the nonlinear $\left(\mathbb{F}_{q}\right.$-linear) operator $d=\sqrt[q]{ } \circ \Delta$, and the $\mathbb{F}_{q^{-}}$-linear Frobenius operator $\tau u=u^{q}$. For example, the function $y={ }_{2} F_{1}(a, b ; c ; z)$ is a solution of the equation

$$
(\Delta-[-a])(\Delta-[-b]) y=d(\Delta-[1-c]) y .
$$

Here we touch only a part of Thakur's results (he considered also hypergeometric functions corresponding to other places of $\mathbb{F}_{q}(x)$, a version of (1.5) with parameters from $K$ and its extensions etc).

The Carlitz exponential $e_{C}$ satisfies a much simpler equation of the same kind:

$$
d e_{C}=e_{C},
$$

so that the operator $d$ may be seen as an analog of the derivative. The operator $\tau$ is an analog of the multiplication by $t$, so that $\Delta$ is the counterpart of $t \frac{d}{d t}$.

The same operators appear in the positive characteristic analogs of the canonical commutation relations of quantum mechanics [17, 18. In the analog of the Schrödinger representation we consider, on the Banach space $C_{0}\left(O, \bar{K}_{c}\right)$ of continuous $\mathbb{F}_{q}$-linear functions on $O$, with values from $\bar{K}_{c}$ (with the supremum norm), the "creation and annihilation operators"

$$
a^{+}=\tau-I, \quad a^{-}=d
$$

( $I$ is the identity operator). Then

$$
a^{-} a^{+}-a^{+} a^{-}=[1]^{1 / q} I
$$

the operator $a^{+} a^{-}$possesses the orthonormal (in the non-Archimedean sense [34]) eigenbasis $\left\{f_{i}\right\}$

$$
\left(a^{+} a^{-}\right) f_{i}=[i] f_{i}, \quad i=0,1,2, \ldots ;
$$

$a^{+}$and $a^{-}$act upon the basis as follows:

$$
a^{+} f_{i-1}=[i] f_{i}, \quad a^{-} f_{i}=f_{i-1}, i \geq 1 ; a^{-} f_{0}=0 .
$$

Here $\left\{f_{i}\right\}$ is the sequence of normalized Carlitz polynomials

$$
f_{i}(s)=D_{i}^{-1} \prod_{\substack{m \in \mathbb{F}_{q}[x] \\ \operatorname{deg} m<i}}(s-m) \quad(i \geq 1), \quad f_{0}(s)=s,
$$


which forms an orthonormal basis in $C_{0}\left(O, \bar{K}_{c}\right)$. The spectrum of the "number operator" $a^{+} a^{-}$ is the set of elements $[i]$, so that even this notation (proposed by Carlitz in 1935) becomes parallel to the usual quantum mechanical situation.

An analog of the Bargmann-Fock representation is obtained if we consider the operators of almost the same form,

$$
\tilde{a}^{+}=\tau, \quad \tilde{a}^{-}=d
$$

but on the Banach space $H$ of power series $u(t)=\sum_{n=0}^{\infty} a_{n} \frac{t^{q^{n}}}{D_{n}}$ with $a_{n} \in \bar{K}_{c}, a_{n} \rightarrow 0$ as $n \rightarrow \infty$. These new operators satisfy the same relations (1.8)-(1.10), but this time instead of the Carlitz polynomials $f_{n}$ we get the eigenfunctions $\tilde{f}_{n}=\frac{t^{q^{n}}}{D_{n}}$.

The above results motivated the author to begin to develop analysis and theory of differential equations for $\mathbb{F}_{q}$-linear functions over $K$ and $\bar{K}_{c}$, that is for the case which can be seen as a concentrated expression of features specific for the analysis in positive characteristic. This paper is a brief survey of some achievements in this direction. In particular, we consider the counterparts of the basic notions of calculus, analytic theory of differential equations (in the regular case and the case of regular singularity), their applications to some special functions, like the power function, logarithm and polylogarithms, Thakur's hypergeometric function etc. An umbral calculus and a theory of holonomic modules are initiated for this case. Like in the classical situation (see [7]), it is shown that some basic objects of the function field arithmetic generate holonomic modules.

Note that some of the results can be easily extended to the case where the base field is a completion of $\mathbb{F}_{q}(x)$ with respect to a finite place determined by an irreducible polynomial $\pi \in$ $\mathbb{F}_{q}[x]$ (the field $K$ corresponds to $\pi(x)=x$ ); for some details see [23]. The situation is different for the "infinite" place widely used in function field arithmetic (see [39]). In this case some of the basic objects behave in a quite different way - absolute values of the Carlitz factorials $D_{n}$ grow, as $n \rightarrow \infty$, the Carlitz exponential is an entire function, the Carlitz polynomials do not form an orthonormal basis etc. A thorough investigation of properties of the Carlitz differential equations for this situation has not been carried out so far.

\section{Calculus}

2.1. Higher Carlitz operators $\Delta^{(n)}$ are introduced recursively,

$$
\left(\Delta^{(n)} u\right)(t)=\Delta^{(n-1)} u(x t)-x^{q^{n-1}} \Delta^{(n-1)} u(t), \quad n \geq 2 .
$$

For $n=1$, the formula $(2.1)$ coincides with the definition of $\Delta=\Delta^{(1)}$, if we set $\Delta^{(0)}=I$.

The first application of these operators is the reconstruction formula [18] for the coefficients $a_{n}$ of a power series $u \in H$. Note that the classical formula does not make sense here because it contains the expression $u^{(n)}(t) / n$ ! where both the numerator and denominator vanish.

Theorem 2.1. If $u \in H$, then

$$
a_{n}=\lim _{t \rightarrow 0} \frac{\Delta^{(n)} u(t)}{t^{q^{n}}}, \quad n=0,1,2, \ldots
$$


For a continuous non-holomorphic $\mathbb{F}_{q}$-linear function $u$ the behaviour of the functions

$$
\mathfrak{D}^{k} u(t)=t^{-q^{k}} \Delta^{(k)} u(t), \quad t \in O \backslash\{0\},
$$

near the origin measures the smoothness of $u$. We say that $u \in C_{0}^{k+1}\left(O, \bar{K}_{c}\right)$ if $\mathfrak{D}^{k} u$ can be extended to a continuous function on $O$. This includes the case $(k=0)$ of differentiable functions.

The next theorem proved in [18 gives a characterization of the above smoothness in terms of coefficients of the Fourier-Carlitz expansion. It includes, as a particular case $(k=0)$, the characterization of differentiable $\mathbb{F}_{q}$-linear functions obtained by Wagner [43].

Theorem 2.2. A function $u=\sum_{n=0}^{\infty} c_{n} f_{n} \in C_{0}\left(O, \bar{K}_{c}\right)$ belongs to $C_{0}^{k+1}\left(O, \bar{K}_{c}\right)$ if and only if

$$
q^{n q^{k}}\left|c_{n}\right| \rightarrow 0 \quad \text { for } n \rightarrow \infty
$$

In this case

$$
\sup _{t \in O}\left|\mathfrak{D}^{k} u(t)\right|=\sup _{n \geq k} q^{(n-k) q^{k}}\left|c_{n}\right|
$$

For a generalization to some classes of not necessarily $\mathbb{F}_{q}$-linear functions see [45.

Similarly [18, a function $u$ is analytic on the ball $O$ (that is, $u(t)=\sum a_{i} t^{q^{i}}, a_{i} \rightarrow 0$ ) if and only if $q^{\frac{q^{n}}{q-1}}\left|c_{n}\right| \rightarrow 0$, as $n \rightarrow \infty$. A more refined result by Yang [44, useful in many applications, which makes it possible to find an exact domain of analyticity, is as follows (again we consider only $\mathbb{F}_{q^{-}}$-linear functions while in [44] a more general class is studied).

Theorem 2.3 (Yang). A function $u=\sum_{n=0}^{\infty} c_{n} f_{n} \in C_{0}\left(O, \bar{K}_{c}\right)$ is locally analytic if and only if

$$
\gamma=\liminf _{n \rightarrow \infty}\left\{-q^{-n} \log _{q}\left|c_{n}\right|\right\}>0,
$$

and if (2.2) holds, then $u$ is analytic on any ball of the radius $q^{-l}$,

$$
l=\max (0,[-(\log (q-1)+\log \gamma) / \log q]+1) .
$$

2.2. Viewing $d$ as a kind of a derivative, it is natural to introduce an antiderivative $S$ setting $S f=u$ where $u$ is a solution of the equation $d u=f$, with the normalization $u(1)=0$. It is easy to find $S f$ explicitly if $f$ is given by its Fourier-Carlitz expansion (see [18]).

Next, we introduce a Volkenborn-type integral of a function $f \in C_{0}^{1}\left(O, \bar{K}_{c}\right)$ (see [34] for a similar integration theory over $\mathbb{Z}_{p}$ ) setting

$$
\int_{O} f(t) d t \stackrel{\text { def }}{=} \lim _{n \rightarrow \infty} \frac{S f\left(x^{n}\right)}{x^{n}}=(S f)^{\prime}(0) .
$$

The integral is a $\mathbb{F}_{q}$-linear continuous functional on $C_{0}^{1}\left(O, \bar{K}_{c}\right)$,

$$
\int_{O} c f(t) d t=c^{q} \int_{O} f(t) d t, \quad c \in \bar{K}_{c}
$$


possessing the following "invariance" property (related, in contrast to the case of $\mathbf{Z}_{p}$, to the multiplicative structure):

$$
\int_{O} f(x t) d t=x \int_{O} f(t) d t-f^{q}(1) .
$$

Calculating the integrals of some important functions we obtain new relations between them. In addition to the Carlitz exponential $e_{C}$ and the Carlitz polynomials $f_{n}$ (see (1.2) and (1.11)), we mention the Carlitz module function

$$
C_{s}(z)=\sum_{i=0}^{\infty} f_{i}(s) z^{q^{i}}, \quad s \in O,|z|<1 .
$$

Note that if $s \in \mathbb{F}_{q}[x]$, then only the terms with $i \leq \operatorname{deg} s$ are different from zero in (2.3).

We have

$$
\begin{gathered}
\int_{O} t^{q^{n}} d t=-\frac{1}{[n+1]}, \quad n=0,1,2, \ldots ; \\
\int_{O} f_{n}(t) d t=\frac{(-1)^{n+1}}{L_{n+1}}, \quad n=0,1,2, \ldots ; \\
\int_{O} C_{s}(z) d s=\log _{C}(z)-z, \quad z \in K,|z|<1 ; \\
\int_{O} e_{C}(s t) d s=t-e_{C}(t), \quad t \in K,|t|<1 .
\end{gathered}
$$

For the proofs see [18].

\section{Differential Equations for $\mathbb{F}_{q}$-Linear Functions}

3.1. Let us consider function field analogs of linear differential equations with holomorphic or polynomial coefficients. Note that in our situation the meaning of a polynomial coefficient is not a usual multiplication by a polynomial, but the action of a polynomial in the operator $\tau$.

We begin with the regular case and consider an equation (actually, a system)

$$
d y(t)=P(\tau) y(t)+f(t)
$$

where for each $z \in\left(\bar{K}_{c}\right)^{m}, t \in K$,

$$
P(\tau) z=\sum_{k=0}^{\infty} \pi_{k} z^{q^{k}}, \quad f(t)=\sum_{j=0}^{\infty} \varphi_{j} \frac{t^{q^{j}}}{D_{j}}
$$

$\pi_{k}$ are $m \times m$ matrices with elements from $\bar{K}_{c}, \varphi_{j} \in\left(\bar{K}_{c}\right)^{m}$, and it is assumed that the series (3.2) have positive radii of convergence. The action of the operator $\tau$ upon a vector or a matrix is defined component-wise, so that $z^{q^{k}}=\left(z_{1}^{q^{k}}, \ldots, z_{m}^{q^{k}}\right)$ for $z=\left(z_{1}, \ldots, z_{m}\right)$. 
We seek a $\mathbb{F}_{q}$-linear solution of (3.1) on some neighbourhood of the origin, of the form

$$
y(t)=\sum_{i=0}^{\infty} y_{i} \frac{t^{q^{i}}}{D_{i}}, \quad y_{i} \in\left(\bar{K}_{c}\right)^{m},
$$

where $y_{0}$ is a given element, so that the "initial" condition for our situation is

$$
\lim _{t \rightarrow 0} t^{-1} y(t)=y_{0}
$$

The next theorem, proved in [19], is the function field analog of the Cauchy theorem from the classical analytic theory of differential equations.

Theorem 3.1. For any $y_{0} \in\left(\bar{K}_{c}\right)^{m}$ the equation (3.1) has a unique local solution of the form (3.3), which satisfies (3.4), with the series having a positive radius of convergence.

Thus, regular equations with Carlitz derivatives behave more or less as their classical counterparts. The situation is different for singular equations. Let us consider scalar equations of arbitrary order

$$
\sum_{j=0}^{m} A_{j}(\tau) d^{j} u=f
$$

where $f(t)=\sum_{n=0}^{\infty} \varphi_{n} \frac{t^{q^{n}}}{D_{n}}, A_{j}(\tau)$ are power series having (as well as the one for $f$ ) positive radii of convergence.

We investigate formal solutions of (3.5), of the form

$$
u(t)=\sum_{n=0}^{\infty} u_{n} \frac{t^{q^{n}}}{D_{n}}, \quad u_{n} \in \bar{K}_{c} .
$$

One can apply an operator series $A(\tau)=\sum_{k=0}^{\infty} \alpha_{k} \tau^{k}$ (even without assuming its convergence) to a formal series (3.6), setting

$$
\tau^{k} u(t)=\sum_{n=0}^{\infty} u_{n}^{q^{k}}[n+1]^{q^{k-1}} \ldots[n+k] \frac{t^{q^{n+k}}}{D_{n+k}}, \quad k \geq 1,
$$

and

$$
A(\tau) u(t)=\sum_{l=0}^{\infty} \frac{t^{q^{l}}}{D_{l}} \sum_{n+k=l} \alpha_{k} u_{n}^{q^{k}}[n+1]^{q^{k-1}} \ldots[n+k]
$$

where the factor $[n+1]^{q^{k-1}} \ldots[n+k]$ is omitted for $k=0$. These formal manipulations are based on the identity

$$
\tau\left(\frac{t^{q^{i-1}}}{D_{i-1}}\right)=[i] \frac{t^{q^{i}}}{D_{i}} .
$$

Using also the relation

$$
d\left(\frac{t^{q^{i}}}{D_{i}}\right)=\frac{t^{q^{i-1}}}{D_{i-1}}
$$

now we can give a meaning to the notion of a formal solution of the equation (3.5). 
Theorem 3.2. Let $u(t)$ be a formal solution (3.6) of the equation (3.5), where the series for $A_{j}(\tau) z, z \in \bar{K}_{c}$, and $f(t)$, have positive radii of convergence. Then the series (3.6) has a positive radius of convergence.

This result (proved in [19]) is in a strong contrast to the classical theory. Note that in the $p$-adic case a similar phenomenon takes place for equations satisfying certain strong conditions upon zeros of indicial polynomials [1, 8, 27, 35]. In our case such a behavior is proved for any equation, which resembles the (much simpler) case [27] of differential equations over a field of characteristics zero, whose residue field also has characteristic zero.

3.2. The equations (3.1) and (3.5) behave like linear equations, though they are actually only $\mathbb{F}_{q}$-linear. Theorem 3.1 can be extended [22] to the case of strongly nonlinear equations (containing self-compositions $y \circ y \circ \cdots \circ y$ ).

On the other hand, it is natural to consider some equations of this kind in wider classes of $\mathbb{F}_{q}$-linear functions resembling meromorphic functions of a complex variables. The set $\mathcal{R}_{K}$ of locally convergent $\mathbb{F}_{q}$-linear holomorphic functions forms a non-commutative ring with respect to the composition operation (the pointwise multiplication violates the $\mathbb{F}_{q}$-linearity). The noncommutativity of $\mathcal{R}_{K}$ makes the algebraic structures related to Carlitz differential equations much more complicated compared to their classical counterparts. So far their understanding is only at its initial stage. It is known, however, that $\mathcal{R}_{K}$ can be imbedded into a skew field of $\mathbb{F}_{q}$-linear "meromorphic" series containing terms like $t^{q^{-k}}$ (see [22]). A deep investigation of bi-infinite series of this kind convergent on the whole of $\bar{K}_{c}$ has been carried out by Poonen [26].

A specific class of equations with solutions meromorphic in the above sense is the class of scalar Riccati-type equations

$$
d y(t)=\lambda(y \circ y)(t)+(P(\tau) y)(t)+R(t)
$$

where $\lambda \in \bar{K}_{c}$,

$$
(P(\tau) y)(t)=\sum_{k=1}^{\infty} p_{k} y^{q^{k}}(t), \quad R(t)=\sum_{k=0}^{\infty} r_{k} t^{q^{k}}
$$

$p_{k}, r_{k} \in \bar{K}_{c}$ (note that the right-hand side of (3.7) does not contain the linear term). The following theorem is proved in 22 .

Theorem 3.3. If $0<|\lambda| \leq q^{-1 / q^{2}},\left|p_{k}\right| \leq q^{-1 / q^{2}},\left|r_{k}\right| \leq q^{-1 / q^{2}}$ for all $k$, then the equation (3.7) possesses solutions of the form

$$
y(t)=c t^{1 / q}+\sum_{n=0}^{\infty} a_{n} t^{q^{n}}, \quad c, a_{n} \in \bar{K}_{c}, c \neq 0,
$$

where the series converges on the open unit disk $|t|<1$. 


\section{Regular Singularity}

4.1. In analysis over $\mathbb{C}$, a typical class of systems with regular singularity at the origin $\zeta=0$ over $\mathbb{C}$ consists of systems of the form

$$
\zeta y^{\prime}(\zeta)=\left(B+\sum_{k=1}^{\infty} A_{k} \zeta^{k}\right) y(\zeta)
$$

where $B, A_{j}$ are constant matrices, and the series converges on a neighbourhood of the origin. Such a system possesses a fundamental matrix solution of the form $W(\zeta) \zeta^{C}$ where $W(\zeta)$ is holomorphic on a neighbourhood of zero, $C$ is a constant matrix, $\zeta^{C}=\exp (C \log \zeta)$ is defined by the obvious power series. Under some additional assumptions regarding the eigenvalues of the matrix $B$, one can take $C=B$. For similar results over $\mathbb{C}_{p}$ see 11 .

In order to investigate such a class of equations in the framework of $\mathbb{F}_{q}$-linear analysis over $K$, one has to go beyond the class of locally analytic functions. Instead of power series expansions we can use the expansions in Carlitz polynomials on the compact ring $O \subset K$. The property of local analyticity, if it takes place, can be recovered with the use of Theorem 2.3. Note that our approach would fail if we consider equations over $\bar{K}_{c}$ instead of $K$ (our solutions may take their values from $\bar{K}_{c}$, but they are defined over subsets of $K$ ). In this sense our techniques are different from the ones developed for both the characteristic zero cases.

We begin with the simplest model scalar equation

$$
\tau d u=\lambda u, \quad \lambda \in \bar{K}_{c}
$$

whose solution may be seen as a function field counterpart of the power function $t \mapsto t^{\lambda}$.

We look for a continuous $\mathbb{F}_{q}$-linear solution $u(t, \lambda)$ of the equation $(4.2)$, with the "initial condition" $u(1, \lambda)=1$, in the form

$$
u(t)=\sum_{i=0}^{\infty} c_{i} f_{i}(t), \quad t \in O
$$

where $c_{0}=1$.

It is easy to see that the equation (4.2) has no continuous solutions if $|\lambda| \geq 1$. If $|\lambda|<1$, then the solution $u(t, \lambda)$ is unique, continuous on $O$, and the coefficients from (4.3) have the form

$$
c_{n}=\prod_{j=0}^{n-1}(\lambda-[j]) .
$$

The function $u(t, \lambda)$ is analytic on $O$ if and only if $\lambda=[j]$ for some $j \geq 0$; in this case $u(t, \lambda)=u(t,[j])=t^{q^{j}}$. If $\lambda \neq[j]$ for any integer $j \geq 0$, then $u(t, \lambda)$ is locally analytic on $O$ if and only if $\lambda=-x$, and in that case $u(t,-x)=0$ for $|t| \leq q^{-1}$. The relation

$$
u\left(t^{q^{m}}, \lambda\right)=u\left(t, \lambda^{q^{m}}+[m]\right), \quad t \in O
$$

holds for all $\lambda,|\lambda|<1$, and for all $m=0,1,2, \ldots$. For the proofs see [20]. 
Similarly, if in (4.1) $\lambda=\left(\lambda_{i j}\right)$ is is a $m \times m$ matrix with elements from $\bar{K}_{c}$, and we look for a matrix-valued solution of (4.1), then such a solution is given by the series (4.3) with the matrix coefficients

$$
c_{i}=\left\{\prod_{j=0}^{i-1}\left(\lambda-[j] I_{m}\right)\right\} c_{0}, \quad i \geq 1
$$

( $I_{m}$ is the unit matrix), if $|\lambda| \stackrel{\text { def }}{=} \max \left|\lambda_{i j}\right|<1$.

4.2. The analog, for our situation, of the system (4.1) is the system

$$
\tau d u-P(\tau) u=0
$$

where $P(\tau)$ is a matrix-valued analytic function, so that $P(\tau) z=\sum_{k=0}^{\infty} \pi_{k} z^{q^{k}}$. We assume that $\left|\pi_{k}\right| \leq \gamma, \gamma>0$, for all $k,\left|\pi_{0}\right|<1$. Denote by $g(t)$ a solution of the equation $\tau d g=\pi_{0} g$. Let $\lambda_{1}, \ldots, \lambda_{m} \in \bar{K}_{c}$ be the eigenvalues of the matrix $\pi_{0}$.

Theorem 4.1. If

$$
\lambda_{i}-\lambda_{j}^{q^{k}} \neq[k], \quad i, j=1, \ldots, m ; k=1,2, \ldots,
$$

then the system (4.4) has a matrix solution

$$
u(t)=W(g(t)), \quad W(s)=\sum_{k=0}^{\infty} w_{k} s^{q^{k}}, \quad w_{0}=I_{m},
$$

where the series for $W$ has a positive radius of convergence.

The paper 20] contains, apart from the proof of Theorem 4.1, a discussion of some situations (the Euler type equations) where its conditions are violated, as well as of the meaning of the conditions (4.5). Here we only mention that in the scalar case $m=1$ the condition (4.5) is equivalent to the assumption $\pi_{0} \neq-x$, so that it excludes the case where solutions of the equation $\tau d g=\pi_{0} g$ has pathological properties.

4.3. For the above equation, continuous solutions were found as Fourier-Carlitz expansions

$$
u(t)=\sum_{n=0}^{\infty} c_{n} f_{n}(t)
$$

and we had to impose certain conditions upon coefficients of the equation, in order to guarantee the uniform convergence of the series on $O$ (which is equivalent to the fact that $c_{n} \rightarrow 0$ ). However formally we could write the series (4.6) for the solutions without those conditions. Thus, it is natural to ask whether the corresponding series (4.6) converge at some points $t \in O$. Note that (4.6) always makes sense for $t \in \mathbb{F}_{q}[x]$ (for each such $t$ only a finite number of terms is different from zero). The question is whether the series converges on a wider set; if the answer is negative, such a formal solution is called strongly singular.

The available results regarding strong singularity of solutions of some equations are based on the following general fact [20]. 
Theorem 4.2. If $\left|c_{i}\right| \geq \rho>0$ for all $i \geq i_{0}$ (where $i_{0}$ is some natural number), then the function (4.6) is strongly singular.

It follows from Theorem 4.2 that non-trivial formal solutions of the equation (4.2) with $|\lambda| \geq 1$ are strongly singular. A more complicated example is provided by the equation

$$
(\Delta-[-a])(\Delta-[-b]) u=d \Delta u, \quad a, b \in \mathbb{Z},
$$

for Thakur's hypergeometric function ${ }_{2} F_{1}(a, b ; 1 ; t)$.

A holomorphic solution of (4.7) is given by an appropriate specialization of (1.5). Classically (over $\mathbb{C}$ ), there exists the second solution with a logarithmic singularity. Here the situation is different. Looking for a solution of the form (4.6) we obtain a recursive relation

$$
\begin{aligned}
\left(c_{i+2}^{1 / q}-c_{i+2}\right)+c_{i+1}^{1 / q}[i+1]^{1 / q}-c_{i+1}([i] & +[i+1]-[-a]-[-b]) \\
& -c_{i}([i]-[-a])([i]-[-b])=0, \quad i=0,1,2, \ldots .
\end{aligned}
$$

Taking arbitrary initial coefficients $c_{0}, c_{1} \in \bar{K}_{c}$ we obtain a solution $u$ defined on $\mathbb{F}_{q}[x]$. On each step we have to solve the equation

$$
z^{1 / q}-z=v
$$

If $\left|c_{i}\right| \leq 1$ and $\left|c_{i+1}\right| \leq 1$, then in the equation for $c_{i+2}$ we have $|v|<1$.

It can be shown [20] that the equation (4.9) has a unique solution $z_{0} \in \bar{K}_{c}$, for which $\left|z_{0}\right| \leq|v|$, and $q-1$ other solutions $z,|z|=1$. It is natural to call a solution generic if, starting from a certain step of finding the coefficients $c_{n}$, we always take the most frequent option corresponding to a solution of (4.9) with $|z|=1$. Now Theorem 4.2 implies the following fact.

Theorem 4.3. A generic solution of the equation (4.7) is strongly singular.

Of course, in some special cases the recursion (4.8) can lead to more regular solutions, in particular, to the holomorphic solutions found by Thakur.

\section{Polylogarithms and a Zeta Function}

5.1. The Carlitz differential equations can be used for defining new special functions with interesting properties. Some examples are given in this section.

An analog of the function $-\log (1-t)$ is defined via the equation

$$
(1-\tau) d u(t)=t, \quad t \in K_{\pi},
$$

a counterpart of the classical equation $(1-t) u^{\prime}(t)=1$. The next results are taken from [23] where the equation (5.1) is considered for an arbitrary finite place of $\mathbb{F}_{q}(x)$.

Let $l_{1}(t)$ be a $\mathbb{F}_{q}$-linear holomorphic solution of (5.1) with the zero initial condition (in the sense of (3.4)). Then it is easy to show that

$$
l_{1}(t)=\sum_{n=1}^{\infty} \frac{t^{q^{n}}}{[n]},
$$


and the series in (5.2) converges for $|t| \leq q^{-1}$.

Note that $l_{1}(t)$ is different from the well-known Carlitz logarithm $\log _{C}$ (see (1.3)). Analogies motivating the introduction of special functions are not so unambiguous, and, for instance, from the composition ring viewpoint, $\log _{C}$ is an analog of $e^{-t}$, though in other respects it is a valuable analog of the logarithm. By the way, another possible analog of the logarithm is a continuous function $u(t),|t|_{\pi} \leq 1$, satisfying the equation $\Delta u(t)=t$ (an analog of $t u^{\prime}(t)=1$ ) and the condition $u(1)=0$. In fact, $u=\mathcal{D}_{1}$, the first hyperdifferential operator (the definition of $\mathcal{D}_{1}$ is given in Sect. 5.2 below); see [16].

Now we consider continuous non-holomorphic extensions of $l_{1}$.

Theorem 5.1. The equation (5.1) has exactly $q$ continuous solutions on $O$ coinciding with (5.2) as $|t| \leq q^{-1}$. These solutions have the expansions in the Carlitz polynomials $u=\sum_{i=0}^{\infty} c_{i} f_{i}$ where $c_{1}$ is an arbitrary solution of the equation $c_{1}^{q}-c_{1}+1=0$, higher coefficients are found from the relation

$$
c_{n}=\sum_{j=0}^{\infty}\left(c_{n-1}[n-1]\right)^{q^{j+1}}, \quad n \geq 2,
$$

and the coefficient $c_{0}$ is determined by the relation

$$
c_{0}=\sum_{i=1}^{\infty}(-1)^{i+1} \frac{c_{i}}{L_{i}},
$$

Below we denote by $l_{1}$ an arbitrary fixed "branch" of extensions of (5.2).

The polylogarithms $l_{n}(t)$ are defined recursively by the equations

$$
\Delta l_{n}=l_{n-1}, \quad n \geq 2
$$

which agree with the classical ones $t l_{n}^{\prime}(t)=l_{n-1}(t)$. Analytic $\mathbb{F}_{q}$-linear solutions of (5.3), such that $t^{-1} l_{n}(t) \rightarrow 0$ as $t \rightarrow 0$, are found easily by induction:

$$
l_{n}(t)=\sum_{j=1}^{\infty} \frac{t^{q^{j}}}{[j]^{n}}, \quad|t| \leq q^{-1}
$$

Theorem 5.2. For each $n \geq 2$, there exists a unique continuous $\mathbb{F}_{q}$-linear solution of the equation (5.3) coinciding for $|t| \leq q^{-1}$ with the polylogarithm (5.4). The solution is given by the Carlitz expansion $l_{n}=\sum_{i=0}^{\infty} c_{i}^{(n)} f_{i}$ with

$$
\begin{gathered}
\left|c_{i}^{(n)}\right| \leq C_{n} q^{-q^{i-1}}, \quad C_{n}>0, i \geq 1, \\
c_{0}^{(n)}=\sum_{i=1}^{\infty}(-1)^{i+1} \frac{c_{i}^{(n)}}{L_{i}},
\end{gathered}
$$


5.2. Now that the above polylogarithms have been extended onto the disk $\{|t| \leq 1\}$, we can interpret their values at $t=1$ as "special values" of a kind of a zeta function. In order to define the latter, we introduce the operator $\Delta^{(\alpha)}, \alpha \in O$, a function field analog of the Hadamard fractional derivative $\left(t \frac{d}{d t}\right)^{\alpha}$ from real analysis (see [33]).

Denote by $\mathcal{D}_{k}(t), k \geq 0, t \in O$, the sequence of hyperdifferentiations defined initially on monomials by the relations $\mathcal{D}_{0}\left(x^{n}\right)=x^{n}, \mathcal{D}_{k}(1)=0, k \geq 1$,

$$
\mathcal{D}_{k}\left(x^{n}\right)=\left(\begin{array}{l}
n \\
k
\end{array}\right) x^{n-k},
$$

where it is assumed that $\left(\begin{array}{l}n \\ k\end{array}\right)=0$ for $k>n$. $\mathcal{D}_{k}$ is extended onto $\mathbb{F}_{q}[x]$ by $\mathbb{F}_{q}$-linearity, and then onto $O$ by continuity [42. The sequence $\left\{\mathcal{D}_{k}\right\}$ is an orthonormal basis of the space of continuous $\mathbb{F}_{q}$-linear functions on $O$ [16, 9].

Let $\alpha \in O, \alpha=\sum_{n=0}^{\infty} \alpha_{n} x^{n}, \alpha_{n} \in \mathbb{F}_{q}$. Denote $\widehat{\alpha}=\sum_{n=0}^{\infty}(-1)^{n} \alpha_{n} x^{n}$. For an arbitrary continuous $\mathbb{F}_{q}$-linear function $u$ on $O$ we define its "fractional derivative" $\Delta^{(\alpha)} u$ at a point $t \in O$ by the formula

$$
\left(\Delta^{(\alpha)} u\right)(t)=\sum_{k=0}^{\infty}(-1)^{k} \mathcal{D}_{k}(\widehat{\alpha}) u\left(x^{k} t\right) .
$$

The function $\alpha \mapsto\left(\Delta^{(\alpha)} u\right)(t)$ is continuous and $\mathbb{F}_{q^{-}}$linear. As a function of $t, \Delta^{(\alpha)} u$ is continuous if, for example, $u$ is Hölder continuous.

Our understanding of $\Delta^{(\alpha)}$ as a kind of a fractional derivative is justified by the following properties:

$$
\begin{gathered}
\Delta^{\left(x^{n}\right)}=\Delta^{n}, \quad n=1,2, \ldots ; \\
\Delta^{(\alpha)}\left(\Delta^{(\beta)} u\right)(t)=\left(\Delta^{(\alpha \beta)} u\right)(t),
\end{gathered}
$$

for any $\alpha, \beta \in O$.

5.3. We define $\zeta(t), t \in K$, setting $\zeta(0)=0$,

$$
\zeta\left(x^{-n}\right)=l_{n}(1), \quad n=1,2, \ldots
$$

and

$$
\zeta(t)=\left(\Delta^{\left(\theta_{0}+\theta_{1} x+\cdots\right)} l_{n}\right)(1), \quad n=1,2, \ldots,
$$

if $t=x^{-n}\left(\theta_{0}+\theta_{1} x+\cdots\right), \theta_{j} \in \mathbb{F}_{q}$. The function $\zeta$ is a continuous $\mathbb{F}_{q}$-linear function on $K_{x}$ with values in $\bar{K}_{c}$.

In particular, we have

$$
\zeta\left(x^{m}\right)=\left(\Delta^{m+1} l_{1}\right)(1), \quad m=0,1,2, \ldots
$$

The above definition is of course inspired by the classical polylogarithm relation

$$
\left(z \frac{d}{d z}\right) \sum_{n=1}^{\infty} \frac{z^{n}}{n^{s}}=\sum_{n=1}^{\infty} \frac{z^{n}}{n^{s-1}} .
$$

In contrast to Goss's zeta function defined on natural numbers and interpolated onto $\mathbb{Z}_{p}$ (see [14, 39]), the above $\zeta$ is purely an object of the characteristic $p$ arithmetic. 
Let us write some relations for special values of our $\zeta$; for the details see [23].

As we saw,

$$
\sum_{j=1}^{\infty} \frac{t^{q^{j}}}{[j]^{n}}=\sum_{i=0}^{\infty} \zeta\left(x^{-n+i}\right) \mathcal{D}_{i}(t), \quad|t| \leq q^{-1} .
$$

Next, let us consider the double sequence $A_{n, r} \in K, A_{n, 1}=(-1)^{n-1} L_{n-1}$,

$$
A_{n, r}=(-1)^{n+r} L_{n-1} \sum_{0<i_{1}<\ldots<i_{r-1}<n} \frac{1}{\left[i_{1}\right]\left[i_{2}\right] \ldots\left[i_{r-1}\right]}, \quad r \geq 2 .
$$

These elements appear as the coefficients of the expansion 42 of a hyperdifferentiation $\mathcal{D}_{r}$ in the normalized Carlitz polynomials, as well as in the expression [15] of the operators $\Delta^{(n)}$ from (2.1) via the iterations $\Delta^{r}$. Here we have the identity

$$
\zeta\left(x^{-n}\right)=\sum_{i=1}^{\infty}(-1)^{i+1} L_{i}^{-1} \sum_{r=1}^{i} A_{i, r} \zeta\left(x^{r-n}\right)
$$

which may be seen as a distant relative of Riemann's functional equation for the classical zeta.

Finally, consider the coefficients $c_{i}$ of the Carlitz expansion of $l_{1}$ (see Theorem 5.1). They are expressed via zeta values:

$$
c_{i}=\sum_{r=1}^{i} A_{i, r} \zeta\left(x^{r-1}\right)
$$

By Theorem 5.1, for $i \geq 2$ we have

$$
c_{i}=\sum_{j=0}^{\infty}\left(z_{i}\right)^{q^{j}}, \quad z_{i}=c_{i-1}^{q}[i-1]^{q}
$$

The series in (5.5) may be seen as an analog of $\sum_{j} j^{-z}$. This analogy becomes clearer if, for a fixed $z \in \bar{K}_{c},|z|<1$, we consider the set $S$ of all convergent power series $\sum_{n=1}^{\infty} z^{q^{j n}}$ corresponding to sequences $\left\{j_{n}\right\} \subset \mathbb{N}$. Let us introduce the multiplication $\odot$ in $S$ setting $z^{q^{i}} \odot z^{q^{j}}=z^{q^{i j}}$ and extending the operation distributively (for a similar construction in the framework of $q$-analysis in characteristic 0 see [25]). Denoting by $\prod_{p}^{\odot}$ the product in $\mathrm{S}$ of elements indexed by prime numbers we obtain in a standard way the identity

$$
c_{i}=\prod_{p} \odot \sum_{n=0}^{\infty}\left(z_{i}\right)^{q^{p^{n}}}
$$

(the infinite product is understood as a limit of the partial products in the topology of $\bar{K}_{c}$ ), an analog of the Euler product formula. It would be interesting to study the algebraic structure of $S$ in detail. 


\section{Umbral Calculus}

6.1. Classical umbral calculus [31, 30] is a set of algebraic tools for obtaining, in a unified way, a rich variety of results regarding structure and properties of various polynomial sequences. There exists a lot of generalizations extending umbral methods to other classes of functions. However there is a restriction common to the whole literature on umbral calculus - the underlying field must be of zero characteristic. An attempt to mimic the characteristic zero procedures in the positive characteristic case [12 revealed a number of pathological properties of the resulting structures. More importantly, these structures were not connected with the existing analysis in positive characteristic based on a completely different algebraic foundation.

A version of umbral calculus inmplementing such a connection was developed by the author [21, and we summarize it in this section. Its basic notion is motivated by the following identity for the non-normalized Carlitz polynomials $e_{i}=D_{i} f_{i}$ :

$$
e_{i}(s t)=\sum_{n=0}^{i}\left(\begin{array}{l}
i \\
n
\end{array}\right)_{K} e_{n}(t)\left\{e_{i-n}(s)\right\}^{q^{n}}
$$

where the "K-binomial coefficients" $\left(\begin{array}{l}i \\ n\end{array}\right)_{K}$ are defined as

$$
\left(\begin{array}{c}
i \\
n
\end{array}\right)_{K}=\frac{D_{i}}{D_{n} D_{i-n}^{q^{n}}}
$$

Computing the absolute values of the Carlitz factorials directly from their definition (1.1), it is easy to show that

$$
\left|\left(\begin{array}{l}
i \\
n
\end{array}\right)_{K}\right|=1, \quad 0 \leq n \leq i
$$

In fact, $\left(\begin{array}{l}i \\ n\end{array}\right)_{K} \in \mathbb{F}_{q}(x)$, and we can consider also other places of $\mathbb{F}_{q}(x)$, that is other nonequivalent absolute values. It can be proved [24] that $\left(\begin{array}{l}i \\ n\end{array}\right)_{K}$ belongs to the ring of integers for any finite place of $\mathbb{F}_{q}(x)$.

We see the relation (6.1) as a function field counterpart of the classical binomial identity 31, 30. satisfied by many classical polynomials. Now, considering a sequence $u_{i}$ of $\mathbb{F}_{q}$-linear polynomials with coefficients from $\bar{K}_{c}$, we call it a sequence of $K$-binomial type if $\operatorname{deg} u_{i}=q^{i}$ and for all $i=0,1,2, \ldots$

$$
u_{i}(s t)=\sum_{n=0}^{i}\left(\begin{array}{l}
i \\
n
\end{array}\right)_{K} u_{n}(t)\left\{u_{i-n}(s)\right\}^{q^{n}}, \quad s, t \in K .
$$

As in the conventional umbral calculus, the dual notion is that of a delta operator. However, in contrast to the classical situation, here the delta operators are only $\mathbb{F}_{q}$-linear, not linear.

Denote by $\rho_{\lambda}$ the operator of multiplicative shift, $\left(\rho_{\lambda} u\right)(t)=u(\lambda t)$. We call a linear operator $T$, on the $\bar{K}_{c}$-vector space $\bar{K}_{c}\{t\}$ of all $\mathbb{F}_{q^{\text {-linear }}}$ polynomials, invariant if it commutes with $\rho_{\lambda}$ for each $\lambda \in K$. 
A $\mathbb{F}_{q}$-linear operator $\delta=\tau^{-1} \delta_{0}$, where $\delta_{0}$ is a linear invariant operator on $\bar{K}_{c}\{t\}$, is called a delta operator if $\delta_{0}(t)=0$ and $\delta_{0}(f) \neq 0$ for $\operatorname{deg} f>1$. A sequence $\left\{P_{n}\right\}_{0}^{\infty}$ of $\mathbb{F}_{q^{-} \text {-linear }}$ polynomials is called a basic sequence corresponding to a delta operator $\delta=\tau^{-1} \delta_{0}$, if $\operatorname{deg} P_{n}=$ $q^{n}, P_{0}(1)=1, P_{n}(1)=0$ for $n \geq 1$,

$$
\delta P_{0}=0, \quad \delta P_{n}=[n]^{1 / q} P_{n-1}, n \geq 1,
$$

or, equivalently,

$$
\delta_{0} P_{0}=0, \quad \delta_{0} P_{n}=[n] P_{n-1}^{q}, n \geq 1 .
$$

It is clear that $d=\tau^{-1} \Delta$ is a delta operator. It follows from well-known identities for the Carlitz polynomials $e_{i}$ [13] (see also (1.10)) that the sequence $\left\{e_{i}\right\}$ is basic with respect to the operator $d$.

Theorem 6.1. For any delta operator $\delta=\tau^{-1} \delta_{0}$, there exists a unique basic sequence $\left\{P_{n}\right\}$, which is a sequence of $K$-binomial type. Conversely, given a sequence $\left\{P_{n}\right\}$ of $K$-binomial type, define the action of $\delta_{0}$ on $P_{n}$ by the relations (6.4), extend it onto $\bar{K}_{c}\{t\}$ by linearity and set $\delta=\tau^{-1} \delta_{0}$. Then $\delta$ is a delta operator, and $\left\{P_{n}\right\}$ is the corresponding basic sequence.

The analogs of the higher Carlitz difference operators (2.1) in the present general context are the operators $\delta_{0}^{(l)}=\tau^{l} \delta^{l}$. The identity

$$
\delta_{0}^{(l)} P_{j}=\frac{D_{j}}{D_{j-l}^{q^{l}}} P_{j-l}^{q^{l}}
$$

holds for any $l \leq j$. If $f$ is a $\mathbb{F}_{q^{-}}$-linear polynomial, $\operatorname{deg} f \leq q^{n}$, then a generalized Taylor formula

$$
f(s t)=\sum_{l=0}^{n} \frac{\left(\delta_{0}^{(l)} f\right)(s)}{D_{l}} P_{l}(t)
$$

holds for any $s, t \in K$. For the Carlitz polynomials $e_{i}$, the formulas (6.5) and (6.6) are well known [13. It is important that, in contrast to the classical umbral calculus, the linear operators involved in (6.6) are not powers of a single linear operator.

Any linear invariant operator $T$ on $\bar{K}_{c}\{t\}$ admits a representation

$$
T=\sum_{l=0}^{\infty} \sigma_{l} \delta_{0}^{(l)}, \quad \sigma_{l}=\frac{\left(T P_{l}\right)(1)}{D_{l}}
$$

The infinite series in (6.7) becomes actually a finite sum if both sides of (6.7) are applied to any $\mathbb{F}_{q}$-linear polynomial. Conversely, any such series defines a linear invariant operator on $\bar{K}_{c}\{t\}$.

Let us consider the case where $\delta=d$, so that $\delta_{0}^{(l)}=\Delta^{(l)}$. The next result leads to new delta operators and basic sequences.

Theorem 6.2. The operator $\theta=\tau^{-1} \theta_{0}$, where

$$
\theta_{0}=\sum_{l=1}^{\infty} \sigma_{l} \Delta^{(l)}
$$


is a delta operator if and only if

$$
S_{n} \stackrel{\text { def }}{=} \sum_{l=1}^{n} \frac{\sigma_{l}}{D_{n-l}^{q^{l}}} \neq 0 \text { for all } n=1,2, \ldots
$$

Example 1. Let $\sigma_{l}=1$ for all $l \geq 1$, that is

$$
\theta_{0}=\sum_{l=1}^{\infty} \Delta^{(l)}
$$

Estimates of $\left|D_{n}\right|$ which follow directly from (1.1) show that $\left|S_{n}\right|=q^{\frac{q^{n}-q}{q-1}}$, so that (6.8) is satisfied. Comparing (6.9) with a classical formula from [31] we may see the polynomials $P_{n}$ for this case as analogs of the Laguerre polynomials.

Example 2. Let $\sigma_{l}=\frac{(-1)^{l+1}}{L_{l}}$. For this case it can be shown [21] that $S_{n}=D_{n}^{-1}, n=$ $1,2, \ldots ; \theta_{0}\left(t^{q^{j}}\right)=t^{q^{j}}$ for all $j \geq 1$ (of course, $\theta_{0}(t)=0$ ), and $P_{0}(t)=t, P_{n}(t)=D_{n}\left(t^{q^{n}}-t^{q^{n-1}}\right)$ for $n \geq 1$.

6.2. As in the $p$-adic case [40, 41, 29], the umbral calculus can be used for constructing new orthonormal bases in $C_{0}\left(O, \bar{K}_{c}\right)$.

Let $\left\{P_{n}\right\}$ be the basic sequence corresponding to a delta operator $\delta=\tau^{-1} \delta_{0}$,

$$
\delta_{0}=\sum_{l=1}^{\infty} \sigma_{l} \Delta^{(l)} .
$$

The sequence $Q_{n}=\frac{P_{n}}{D_{n}}, n=0,1,2, \ldots$, called the normalized basic sequence, satisfies the identity

$$
Q_{i}(s t)=\sum_{n=0}^{i} Q_{n}(t)\left\{Q_{i-n}(s)\right\}^{q^{n}},
$$

another form of the $K$-binomial property. Though it resembles its classical counterpart, the presence of the Frobenius powers is a feature specific for the case of a positive characteristic.

Theorem 6.3. If $\left|\sigma_{1}\right|=1,\left|\sigma_{l}\right| \leq 1$ for $l \geq 2$, then the sequence $\left\{Q_{n}\right\}_{0}^{\infty}$ is an orthonormal basis of the space $C_{0}\left(O, \bar{K}_{c}\right)$ - for any $f \in C_{0}\left(O, \bar{K}_{c}\right)$ there is a uniformly convergent expansion

$$
f(t)=\sum_{n=0}^{\infty} \psi_{n} Q_{n}(t), \quad t \in O,
$$

where $\psi_{n}=\left(\delta_{0}^{(n)} f\right)(1),\left|\psi_{n}\right| \rightarrow 0$ as $n \rightarrow \infty$,

$$
\|f\|=\sup _{n \geq 0}\left|\psi_{n}\right| .
$$


By Theorem 6.3, the Laguerre-type polynomial sequence from Example 1 is an orthonormal basis of $C_{0}\left(O, \bar{K}_{c}\right)$. The sequence from Example 2 does not satisfy the conditions of Theorem 6.3 .

Note that the conditions of Theorem 6.3 imply that $S_{n} \neq 0$ for all $n$, so that the series (6.10) considered in Theorem 6.3 always correspond to delta operators.

In 21] recursive formulas and generating functions for normalized basic sequences are also given.

\section{The Weyl-Carlitz Ring and Holonomic Modules}

7.1. The theory of holonomic modules over the Weyl algebra and more general algebras of differential or $q$-difference operators is becoming increasingly important, both as a crucial part of the general theory of D-modules and in view of various applications (see, for example, 4, 4, 32 ). Usually, the holonomic property of the module corresponding to a system of differential equations is a sign of its "regular" behavior. Most of the classical special functions are associated (see [7) with holonomic modules, which helps to investigate their properties.

It is clear from the above results that in the positive characteristic case a natural counterpart of the Weyl algebra is, for the case of a single variable, the ring $\mathfrak{A}_{1}$ generated by $\tau$, $d$, and scalars from $\bar{K}_{c}$, with the relations

$$
d \tau-\tau d=[1]^{1 / q}, \quad \tau \lambda=\lambda^{q} \tau, \quad d \lambda=\lambda^{1 / q} d\left(\lambda \in \bar{K}_{c}\right)
$$

The ring consists of finite sums

$$
a=\sum_{i, j} \lambda_{i j} \tau^{i} d^{j}, \quad \lambda_{i j} \in \bar{K}_{c}
$$

and the representation of an element in the form (7.2) is unique.

Basic algebraic properties of $\mathfrak{A}_{1}$ [19, 3] are similar to those of the Weyl algebra in characteristic 0 and quite different from the case of the algebra of usual differential operators over a field of positive characteristic [28].

The ring $\mathfrak{A}_{1}$ is left and right Noetherian, without zero divisors. $\mathfrak{A}_{1}$ possesses no non-trivial two-sided ideals stable with respect to the mapping

$$
\sum_{i, j} \lambda_{i j} \tau^{i} d^{j} \mapsto \sum_{i, j} \lambda_{i j}^{q} \tau^{i} d^{j}
$$

The centre of $\mathfrak{A}_{1}$ is described explicitly in [3]; it contains countably many elements (this corrects an erroneous statement from [19]). In fact, $\mathfrak{A}_{1}$ belongs to the class of generalized Weyl algebras 2]. A well-developed theory available for them enabled Bavula [3] to classify ideals in $\mathfrak{A}_{1}$, as well as all simple modules over $\mathfrak{A}_{1}$.

A generalization of $\mathfrak{A}_{1}$ to the case of several variables is not straightforward because the Carlitz derivatives $d_{s}$ and $d_{t}$ do not commute on a monomial $f(s, t)=s^{q^{m}} t^{q^{n}}$, if $m \neq n$.

Moreover, if $m>n$, then $d_{s}^{m} f$ is not a polynomial, nor even a holomorphic function in $t$ (since the action of $d$ is not linear and involves taking the $q$-th root). 
A reasonable generalization is inspired by Zeilberger's idea (see [7]) to study holonomic properties of sequences of functions making a transform with respect to the discrete variables, which reduces the continuous-discrete case to the purely continuous one (simultaneously in all the variables). In our situation, if $\left\{P_{k}(s)\right\}$ is a sequence of $\mathbb{F}_{q}$-linear polynomials with $\operatorname{deg} P_{k} \leq q^{k}$, we set

$$
f(s, t)=\sum_{k=0}^{\infty} P_{k}(s) t^{q^{k}}
$$

and $d_{s}$ is well-defined. In the variable $t$, we consider not $d_{t}$ but the linear operator $\Delta_{t}$. The latter does not commute with $d_{s}$ either, but satisfies the commutation relations

$$
d_{s} \Delta_{t}-\Delta_{t} d_{s}=[1]^{1 / q} d_{s}, \quad \Delta_{t} \tau-\tau \Delta_{t}=[1] \tau,
$$

so that the resulting ring $\mathfrak{A}_{2}$ resembles a universal enveloping algebra of a solvable Lie algebra.

More generally, denote by $\mathcal{F}_{n+1}$ the set of all germs of functions of the form

$$
f\left(s, t_{1}, \ldots, t_{n}\right)=\sum_{k_{1}=0}^{\infty} \ldots \sum_{k_{n}=0}^{\infty} \sum_{m=0}^{\min \left(k_{1}, \ldots, k_{n}\right)} a_{m, k_{1}, \ldots, k_{n}} s^{q^{m}} t_{1}^{q^{k_{1}}} \ldots t_{n}^{q^{k_{n}}}
$$

where $a_{m, k_{1}, \ldots, k_{n}} \in \bar{K}_{c}$ are such that all the series are convergent on some neighbourhoods of the origin. We do not exclude the case $n=0$ where $\mathcal{F}_{1}$ will mean the set of all $\mathbb{F}_{q}$-linear power series $\sum_{m} a_{m} s^{q^{m}}$ convergent on a neighbourhood of the origin. $\widehat{\mathcal{F}}_{n+1}$ will denote the set of all polynomials from $\mathcal{F}_{n+1}$, that is the series (7.4) in which only a finite number of coefficients is different from zero.

The ring $\mathfrak{A}_{n+1}$ is generated by the operators $\tau, d_{s}, \Delta_{t_{1}}, \ldots \Delta_{t_{n}}$ on $\mathcal{F}_{n+1}$, and the operators of multiplication by scalars from $\bar{K}_{c}$. To simplify the notation, we write $\Delta_{j}$ instead of $\Delta_{t_{j}}$ and identify a scalar $\lambda \in \bar{K}_{c}$ with the operator of multiplication by $\lambda$. The operators $\Delta_{j}$ are $\bar{K}_{c}$-linear, so that

$$
\Delta_{j} \lambda=\lambda \Delta_{j}, \quad \lambda \in \bar{K}_{c},
$$

while the operators $\tau, d_{s}$ satisfy the commutation relations (7.1). In the action of each operator $d_{s}, \Delta_{j}$ (acting in a single variable), other variables are treated as scalars. The operator $\tau$ acts simultaneously on all the variables and coefficients. We have the relations involving $\Delta_{j}$ :

$$
\Delta_{j} \tau-\tau \Delta_{j}=[1] \tau, \quad d_{s} \Delta_{j}-\Delta_{j} d_{s}=[1]^{1 / q} d_{s}, \quad j=1, \ldots, n .
$$

Using the commutation relations (7.1), (7.5), and (7.6), we can write any $a \in \mathfrak{A}_{n+1}$, in a unique way, as a finite sum

$$
a=\sum c_{l, \mu, i_{1}, \ldots, i_{n}} \tau^{l} d_{s}^{\mu} \Delta_{1}^{i_{1}} \ldots \Delta_{n}^{i_{n}}
$$

Let us introduce a filtration in $\mathfrak{A}_{n+1}$ (an analog of the Bernstein filtration) denoting by $\Gamma_{\nu}$,

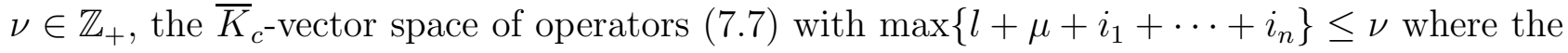
maximum is taken over all the terms of (7.7). Then $\mathfrak{A}_{n+1}$ is a left and right Noetherian filtered ring. 
In a standard way (see [10]) we define filtered left modules over $\mathfrak{A}_{n+1}$. All the basic notions regarding a filtered module $M$ (like those of the graded module $\operatorname{gr}(M)$, dimension $d(M)$, multiplicity $m(M)$, good filtration etc) are introduced just as their counterparts in the theory of modules over the Weyl algebra.

If we consider $\mathfrak{A}_{n+1}$ as a left module over itself, then

$$
d\left(\mathfrak{A}_{n+1}\right)=n+2, \quad m\left(\mathfrak{A}_{n+1}\right)=1 .
$$

For any finitely generated left $\mathfrak{A}_{n+1}$-module $M$, we have $d(M) \leq n+2$. By (7.8), this bound cannot be improved in general. However, if $I$ is a non-zero left ideal in $\mathfrak{A}_{n+1}$, then

$$
d\left(\mathfrak{A}_{n+1} / I\right) \leq n+1
$$

For the module $\widehat{\mathcal{F}}_{n+1}$ of $\mathbb{F}_{q^{-}}$-linear polynomials (7.4), we have

$$
d\left(\widehat{\mathcal{F}}_{n+1}\right)=n+1, \quad m\left(\widehat{\mathcal{F}}_{n+1}\right)=n !
$$

The proofs of all these results, as well as the ones given in this section below, can be found in 24 .

It is natural to call an $\mathfrak{A}_{n+1}$-module $M$ holonomic if $d(M)=n+1$. Thus, $\widehat{\mathcal{F}}_{n+1}$ is an example of a holonomic module.

The next theorem demonstrates, already for the case of $\mathfrak{A}_{1}$-modules, a sharp difference from the case of modules over the Weyl algebras. In particular, we see that an analog of the Bernstein inequality (see [10]) does not hold here without some additional assumptions.

Theorem 7.1. (i) For any $k=1,2, \ldots$, there exists such a nontrivial $\mathfrak{A}_{1}$-module $M$ that $\operatorname{dim} M=k$ (dim means the dimension over $\left.\bar{K}_{c}\right)$, that is $d(M)=0$.

(ii) Let $M$ be a finitely generated $\mathfrak{A}_{1}$-module with a good filtration. Suppose that there exists a "vacuum vector" $v \in M$, such that $d_{s} v=0$ and $\tau^{m}(v) \neq 0$ for all $m=0,1,2, \ldots$ Then $d(M) \geq 1$.

7.3. Let us consider the case of holonomic submodules of the $\mathfrak{A}_{n+1}$-module $\mathcal{F}_{n+1}$, consisting of $\mathbb{F}_{q}$-linear functions (7.4) polynomial in $s$ and holomorphic near the origin in $t_{1}, \ldots, t_{n}$.

Let $0 \neq f \in \mathcal{F}_{n+1}$,

$$
I_{f}=\left\{\varphi \in \mathfrak{A}_{n+1}: \varphi(f)=0\right\} .
$$

$I_{f}$ is a left ideal in $\mathfrak{A}_{n+1}$. The left $\mathfrak{A}_{n+1}$-module $M_{f}=\mathfrak{A}_{n+1} / I_{f}$ is isomorphic to the submodule $\mathfrak{A}_{n+1} f \subset \mathcal{F}_{n+1}-$ an element $\varphi(f) \in \mathfrak{A}_{n+1} f$ corresponds to the class of $\varphi \in \mathfrak{A}_{n+1}$ in $M_{f}$. A natural good filtration in $M_{f}$ is induced from that in $\mathfrak{A}_{n+1}$.

As we know (see (7.9)), if $I_{f} \neq\{0\}$, then $d\left(M_{f}\right) \leq n+1$. We call a function $f$ holonomic if the module $M_{f}$ is holonomic, that is $d\left(M_{f}\right)=n+1$. The condition $I_{f} \neq\{0\}$ means that $f$ is a solution of a non-trivial "differential equation" $\varphi(f)=0, \varphi \in \mathfrak{A}_{n+1}$. The case $n=0$ is quite simple.

Theorem 7.2. If a non-zero function $f \in \mathcal{F}_{1}$ satisfies an equation $\varphi(f)=0,0 \neq \varphi \in \mathfrak{A}_{1}$, then $f$ is holonomic. 
In particular, any $\mathbb{F}_{q}$-linear polynomial of $s$ is holonomic, since it is annihilated by $d_{s}^{m}$, with a sufficiently large $m$.

If $n>0$, the situation is more complicated. We call the module $M_{f}$ (and the corresponding function $f$ ) degenerate if $D\left(M_{f}\right)<n+1$ (by the Bernstein inequality, there is no degeneracy phenomena for modules over the complex Weyl algebra). The simplest example of a degenerate function (for $n=1$ ) is $f\left(s, t_{1}\right)=g\left(s t_{1}\right) \in \mathcal{F}_{2}$ where the function $g$ belongs to $\mathcal{F}_{1}$ and satisfies an equation $\varphi(g)=0, \varphi \in \mathfrak{A}_{1}$. It can be shown that $d\left(M_{f}\right)=1$.

In order to exclude the degenerate case, we introduce the notion of a non-sparse function.

A function $f \in \mathcal{F}_{n+1}$ of the form (7.4) is called non-sparse if there exists such a sequence $m_{l} \rightarrow \infty$ that, for any $l$, there exist sequences $k_{1}^{(i)}, k_{2}^{(i)}, \ldots, k_{n}^{(i)} \geq m_{l}$ (depending on $l$ ), such that $k_{\nu}^{(i)} \rightarrow \infty$ as $i \rightarrow \infty(\nu=1, \ldots, n)$, and $a_{m, k_{1}^{(i)}, \ldots, k_{n}^{(i)}} \neq 0$.

Theorem 7.3. If a function $f$ is non-sparse, then $d\left(M_{f}\right) \geq n+1$. If, in addition, $f$ satisfies an equation $\varphi(f)=0,0 \neq \varphi \in \mathfrak{A}_{n+1}$, then $f$ is holonomic.

7.4 We use Theorem 7.3 to prove that the functions (7.4) obtained via the sequence-tofunction transform (7.3) or its multi-index generalizations, from some well-known sequences of polynomials over $K$ are holonomic. In all the cases below the non-sparseness is evident, and we have only to prove that the corresponding function satisfies a non-trivial Carlitz differential equation.

a) The Carlitz polynomials. The transform (7.3) of the sequence $\left\{f_{k}\right\}$ is the Carlitz module function $C_{s}(t)$; see (2.3). It is easy to check that $d_{s} C_{s}(t)=C_{s}(t)$. Therefore the Carlitz module function is holonomic, jointly in both its variables.

b) Thakur's hypergeometric polynomials. We consider the polynomial case of Thakur's hypergeometric function (1.5), that is

$$
{ }_{l} F_{\lambda}\left(-a_{1}, \ldots,-a_{l} ;-b_{1}, \ldots,-b_{\lambda} ; z\right)=\sum_{m} \frac{\left(-a_{1}\right)_{m} \ldots\left(-a_{l}\right)_{m}}{\left(-b_{1}\right)_{m} \ldots\left(-b_{\lambda}\right)_{m} D_{m}} z^{q^{m}}
$$

where $a_{1}, \ldots, a_{l}, b_{1}, \ldots, b_{\lambda} \in \mathbb{Z}_{+}$. It is seen from (1.4) that the terms in (7.10), which make sense and do not vanish, are those with $m \leq \min \left(a_{1}, \ldots, a_{l}, b_{1}, \ldots, b_{\lambda}\right)$. Let the function $f \in \mathcal{F}_{l+\lambda+1}$ be given by

$$
\begin{gathered}
f\left(s, t_{1}, \ldots, t_{l}, u_{1}, \ldots, u_{\lambda}\right) \\
=\sum_{k_{1}=0}^{\infty} \ldots \sum_{k_{l}=0}^{\infty} \sum_{\nu_{1}=0}^{\infty} \ldots \sum_{\nu_{\lambda}=0}^{\infty}{ }_{l} F_{\lambda}\left(-k_{1}, \ldots,-k_{l} ;-\nu_{1}, \ldots,-\nu_{\lambda} ; s\right) \\
\times t_{1}^{q^{k_{1}}} \ldots t_{l}^{q^{k_{l}}} u_{1}^{q^{\nu_{1}}} \ldots u_{\lambda}^{q^{\nu_{\lambda}}} .
\end{gathered}
$$

It is known ([39], Sect. 6.5) that

$$
\begin{aligned}
d_{s l} F_{\lambda}\left(-k_{1}, \ldots,-k_{l} ;-\nu_{1}, \ldots,-\nu_{\lambda} ; s\right) & \\
& ={ }_{l} F_{\lambda}\left(-k_{1}+1, \ldots,-k_{l}+1 ;-\nu_{1}+1, \ldots,-\nu_{\lambda}+1 ; s\right)
\end{aligned}
$$


if all the parameters $k_{1}, \ldots, k_{l}, \nu_{1}, \ldots, \nu_{\lambda}$ are different from zero. If at least one of them is equal to zero, then the left-hand side of (7.11) equals zero. This property implies the identity $d_{s} f=f$, the same as that for the Carlitz module function. Thus, $f$ is holonomic.

c). K-binomial coefficients. It can be shown [24] that the $K$-binomial coefficients $\left(\begin{array}{c}k \\ m\end{array}\right)_{K}$ (see Sect. 6) satisfy the Pascal-type identity

$$
\left(\begin{array}{c}
k \\
m
\end{array}\right)_{K}=\left(\begin{array}{c}
k-1 \\
m-1
\end{array}\right)_{K}^{q}+\left(\begin{array}{c}
k-1 \\
m
\end{array}\right)_{K}^{q} D_{m}^{q-1}
$$

where $0 \leq m \leq k$ and it is assumed that $\left(\begin{array}{c}k \\ -1\end{array}\right)_{K}=\left(\begin{array}{c}k-1 \\ k\end{array}\right)_{K}=0$.

Consider a function $f \in \mathcal{F}_{2}$ associated with the $K$-binomial coefficients, that is

$$
f(s, t)=\sum_{k=0}^{\infty} \sum_{m=0}^{k}\left(\begin{array}{c}
k \\
m
\end{array}\right)_{K} s^{q^{m}} t^{q^{k}} .
$$

The identity (7.12) implies the equation

$$
d_{s} f(s, t)=\Delta_{t} f(s, t)+[1]^{1 / q} f(s, t)
$$

for the function (7.13). Therefore $f$ is holonomic.

\section{References}

[1] F. Baldassarri, Differential modules and singular points of $p$-adic differential equations, Adv. Math. 44 (1982), 155-179.

[2] V. Bavula, Generalized Weyl algebras and their representations, St. Petersburg Math. J. 4, No. 1 (1993), 71-92.

[3] V. Bavula, The Carlitz algebras, math.RA/0505397.

[4] Yu. Berest and A. Kasman, D-modules and Darboux transformations, Lett. Math. Phys. 43 (1998), 279-294.

[5] L. Carlitz, On certain functions connected with polynomials in a Galois field, Duke Math. J. 1 (1935), 137-168.

[6] L. Carlitz, Some special functions over $G F(q, x)$, Duke Math. J. 27 (1960), 139-158.

[7] P. Cartier, Démonstration "automatique" d'identités et fonctions hypergéometriques (d'après D. Zeilberger), Astérisque 206 (1992), 41-91.

[8] D. N. Clark, A note on the $p$-adic convergence of solutions of linear differential equations, Proc. Amer. Math. Soc. 17 (1966), 262-269. 
[9] K. Conrad, The digit principle, J. Number Theory 84 (2000), 230-237.

[10] S. C. Coutinho, A Primer of Algebraic D-modules, Cambridge University Press, 1995.

[11] B. Dwork, G. Gerotto, and F. J. Sullivan, An Introduction to G-Functions, Princeton University Press, 1994.

[12] L. Ferrari, An umbral calculus over infinite coefficient fields of positive characteristic, Comp. Math. Appl. 41 (2001), 1099-1108.

[13] D. Goss, Fourier series, measures, and divided power series in the theory of function fields, K-Theory 1 (1989), 533-555.

[14] D. Goss, Basic Structures of Function Field Arithmetic, Springer, Berlin, 1996.

[15] S. Jeong, Continuous linear endomorphisms and difference equations over the completions of $\mathbb{F}_{q}[T]$, J. Number Theory 84 (2000), 276-291.

[16] S. Jeong, Hyperdifferential operators and continuous functions on function fields, J. Number Theory 89 (2001), 165-178.

[17] A. N. Kochubei, Harmonic oscillator in characteristic p, Lett. Math. Phys. 45 (1998), $11-20$.

[18] A. N. Kochubei, $\mathbb{F}_{q}$-linear calculus over function fields, J. Number Theory 76 (1999), 281-300.

[19] A. N. Kochubei, Differential equations for $\mathbb{F}_{q}$-linear functions, J. Number Theory 83 (2000), 137-154.

[20] A. N. Kochubei, Differential equations for $\mathbb{F}_{q}$-linear functions II: Regular singularity, Finite Fields Appl. 9 (2003), 250-266.

[21] A. N. Kochubei, Umbral calculus in positive characteristic, Adv. Appl. Math. 34 (2005), $175-191$.

[22] A. N. Kochubei, Strongly nonlinear differential equations with Carlitz derivatives over a function field, Ukrainian Math. J. 57, No. 5 (2005); math.NT/ 0405542.

[23] A. N. Kochubei, Polylogarithms and a zeta function for finite places of a function field, Contemporary Math. 384 (2005); math.NT/0405544.

[24] A. N. Kochubei, Holonomic modules in positive characteristic, math.RA/0503398.

[25] M. B. Nathanson, Additive number theory and the ring of quantum integers, math.NT/0204006.

[26] B. Poonen, Fractional power series and pairings on Drinfeld modules, J. Amer. Math. Soc. 9 (1996), 783-812. 
[27] M. van der Put, Meromorphic differential equations over valued fields, Indag. Math. 42 (1980), 327-332.

[28] M. van der Put, Differential equations in characteristic p, Compositio Math. 97 (1995), $227-251$.

[29] A. M. Robert, A Course in p-Adic Analysis, Springer, New York, 2000.

[30] S. Roman, The Umbral Calculus, Academic Press, London, 1984.

[31] G.-C. Rota, D. Kahaner and A. Odlyzko, On the foundations of combinatorial theory. VIII. Finite operator calculus, J. Math. Anal. Appl. 42 (1973), 684-760.

[32] C. Sabbah, Systèmes holonomes d'équations aux q-differences. In: D-modules and Microlocal Geometry (M. Kashiwara et al., eds.), Walter de Gruyter, Berlin, 1993, pp. $125-147$.

[33] S. G. Samko, A. A. Kilbas, and O. I. Marichev, Fractional Integrals and Derivatives: Theory and Applications, Gordon and Breach, New York, 1993.

[34] W. Schikhof, Ultrametric Calculus, Cambridge University Press, 1984.

[35] M. Setoyanagi, Note on Clark's theorem for p-adic convergence, Proc. Amer. Math. Soc. 125 (1997), 717-721.

[36] D. Sinnou and D. Laurent, Indépendence algebrique sur les T-modules, Compositio Math. $122(2000), 1-22$.

[37] D. S. Thakur, Hypergeometric functions for function fields, Finite Fields and Their Appl. 1 (1995), 219-231.

[38] D. S. Thakur, Hypergeometric functions for function fields II, J. Ramanujan Math. Soc. 15 (2000), 43-52.

[39] D. S. Thakur, Function Field Arithmetic, World Scientific, Singapore, 2004.

[40] L. Van Hamme, Continuous operators, which commute with translations, on the space of continuous functions on $\mathbb{Z}_{p}$. In: p-Adic Functional Analysis (J. M. Bayod et al., eds.), Lect. Notes Pure Appl. Math. 137, Marcel Dekker, New York, 1992, pp. 75-88.

[41] A. Verdoodt, Umbral calculus in non-Archimedean analysis. In: p-Adic Functional Analysis (A. K. Katsaras et al., eds.), Lect. Notes Pure Appl. Math. 222, Marcel Dekker, New York, 2001, pp. 309-322.

[42] J. F. Voloch, Differential operators and interpolation series in power series fields, $J$. Number Theory 71 (1998), 106-108.

[43] C. G. Wagner, Linear operators in local fields of prime characteristic, J. Reine Angew. Math. 251 (1971), 153-160. 
[44] Z. Yang, Locally analytic functions over completions of $\mathbf{F}_{r}(U)$, J. Number Theory $\mathbf{7 3}$ (1998), 451-458.

[45] Z. Yang, $C^{n}$-functions over completions of $\mathbb{F}_{r}[T]$ at finite places of $\mathbb{F}_{r}(T), J$. Number Theory 108 (2004), 346-374. 